
Not for reproduction, distribution or commercial use.

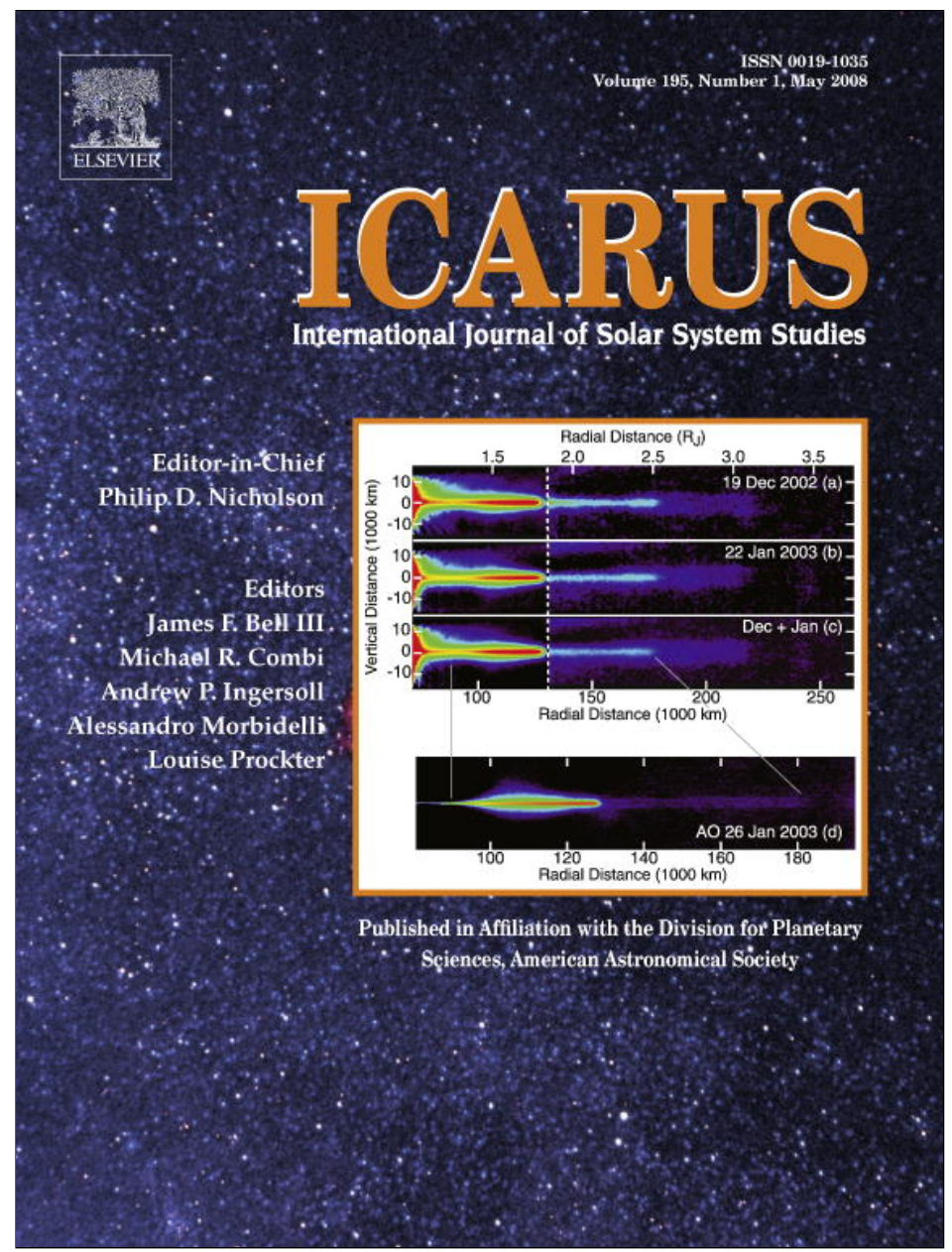

This article appeared in a journal published by Elsevier. The attached copy is furnished to the author for internal non-commercial research and education use, including for instruction at the authors institution and sharing with colleagues.

Other uses, including reproduction and distribution, or selling or licensing copies, or posting to personal, institutional or third party websites are prohibited.

In most cases authors are permitted to post their version of the article (e.g. in Word or Tex form) to their personal website or institutional repository. Authors requiring further information regarding Elsevier's archiving and manuscript policies are encouraged to visit:

http://www.elsevier.com/copyright 


\title{
The librations, shape, and icy shell of Europa
}

\author{
T. Van Hoolst ${ }^{\mathrm{a}, *}$, N. Rambaux ${ }^{\mathrm{a}, \mathrm{b}}$, Ö. Karatekin ${ }^{\mathrm{a}}$, V. Dehant ${ }^{\mathrm{a}}$, A. Rivoldini ${ }^{\mathrm{a}}$ \\ a Royal Observatory of Belgium, Ringlaan 3, B-1180 Brussels, Belgium \\ b Département de Mathématique, Facultés Univ. N.D. de la Paix, 8 Rempart de la Vierge, B-5000 Namur, Belgium \\ Received 20 July 2007; revised 20 November 2007 \\ Available online 10 January 2008
}

\begin{abstract}
Europa, the smallest of the Galilean satellites, has a young icy surface and most likely contains an internal ocean. The primary objective of possible future missions to Europa is the unambiguous detection and characterization of a subsurface ocean. The thickness of the overlying icy shell provides important information on the thermal evolution of the satellite and on the interaction between the ocean and the surface, the latter being fundamental for astrobiology. However, the thickness is not well known, and estimates range from several hundred of meters to some ten of kilometers. Here, we investigate the use of libration (rotation variation) observations to study the interior structure of Europa and in particular its icy shell. A dynamical libration model is developed, which includes gravitational coupling between the icy shell and the heavy solid interior. The amplitude of the main libration signal at 3.55 days (the orbital period) is shown to depend on Europa's shape and structure. Models of the interior structure of Europa are constructed and the equatorial flattening of the internal layers, which are key parameters for the libration, are calculated by assuming that Europa is in hydrostatic equilibrium. Europa's flattened shape is assumed to be due to rotation and permanent tides, and we extend the classical Radau equation for rotationally flattened bodies to include also tidal deformation. We show that the presence of an ocean increases the amplitude of libration by about $10 \%$, depending mainly on the thickness of the icy shell. Therefore, libration observations offer possibility of detection of a subsurface ocean in Europa and estimation of the thickness of its overlying icy shell.
\end{abstract}

(c) 2008 Elsevier Inc. All rights reserved.

Keywords: Europa; Interiors; Rotational dynamics; Spin-orbit resonances; Tides, solid body

\section{Introduction}

Voyager and Galileo images provided the first indications for an internal ocean in Europa (Squyres et al., 1983; Carr et al., 1998; Pappalardo et al., 1999), but the most robust evidence for a subsurface ocean is the detection of an induced magnetic field in the vicinity of Europa by the Galileo mission (Kivelson et al., 2000). This field implies the presence of an electrically conducting internal layer several tens of kilometers thick, which could consist of salty liquid water. A subsurface ocean is also in agreement with theoretical models of the interior structure and thermal evolution of Europa (Ross and Schubert, 1987; Spohn and Schubert, 2003; Sotin et al., 2002; Hussmann et al., 2006).

\footnotetext{
* Corresponding author. Fax: +32 23749822.

E-mail address: tim.vanhoolst@oma.be (T. Van Hoolst).
}

To unambiguously prove the existence of a subsurface ocean and to characterize its depth and the thickness of the overlying icy shell, several measurement techniques have been proposed. Measurements of the time-variable tidal gravity variations through the dynamic effect of Europa's gravity field on the trajectory of an orbiter (Wu et al., 2001), preferably combined with altimetry measurements of the tidal surface displacements (Wahr et al., 2006; Castillo et al., 2000), as well as extensive characterization of the magnetic field in the neighborhood of Europa by a magnetometer on board of a low-flying orbiter (Khurana et al., 1998; Kivelson et al., 1999, 2000) can provide conclusive information for the detection of an ocean. However, tidal measurements may not be sufficient to determine accurately the depth and thickness mainly because of the poorly known composition and rheological characteristics of the icy shell (Wahr et al., 2006), and in interpretations of magnetic data the depth of the ocean may be difficult to separate from the ocean conductivity. A clear picture of Europa's interior could 
be obtained from seismic sounding (Kovach and Chyba, 2001; Lee et al., 2003), but that would require a lander.

Measurements of the librations (rotation variations) of Europa to characterize the putative ocean have been studied by $\mathrm{Wu}$ et al. (2001). However, published models of the librations of Europa assume that Europa responds as a rigid body to the gravitational torque exerted by Jupiter and offer no possibility to deduce information on the ocean (Comstock and Bills, 2003; Henrard, 2005). Here, we take into account that Europa has a differentiated structure with a possible internal ocean and develop a model of libration that includes dynamical coupling between different internal layers. In particular, gravitational coupling (Szeto and Xu, 1997; Buffett, 1996) between the icy shell and the solid interior, which contains most of Europa's mass, is studied and shown to alter significantly the libration amplitude. Our objective is to investigate whether libration observations can be used to determine the existence of a subsurface ocean and the thickness of the icy shell. Provided that the amplitude is large enough, the librational response of Europa can be characterized efficiently from orbit using remote sensing techniques such as a camera and an altimeter. Furthermore, the librations can be obtained as a part of the global time-variable gravity solution of a spacecraft in orbit around Europa (Wu et al., 2001).

The paper is organized as follows. In Section 2, we develop a new model for the libration of Europa that includes the gravitational coupling between the icy shell and the solid interior. The layered internal structure of Europa as well as the surface and internal flattenings necessary for the characterization of the librational response and gravitational coupling are discussed in Section 3. For the construction of the models of interior structure of Europa, we extend the classical Radau equation, which relates the rotational polar flattening to the mean moment of inertia, to synchronously rotating satellites flattened by rotation and permanent tides. The interior structure models are constrained by the total mass, radius, and mean moment of inertia of Europa. The flattenings of the internal layers are calculated by using Clairaut theory. The differences in the librational response of Europa as a function of the presence of a subsurface ocean, the thickness of the icy shell, and the internal coupling are studied in Section 4. Section 5 presents a discussion and conclusion.

\section{Librations}

\subsection{Rigid librations}

Since the synchronously rotating Europa is not spherically symmetric, gravitational torques by other Solar System bodies are exerted on it and can change its rotation. We only consider the gravitational interaction between Jupiter and Europa since the torque of Jupiter is at least three orders of magnitude larger than the torque of any other body (the second-largest torque is due to Io). For the torque calculation, Jupiter is assumed to be spherically symmetric. We also take the orbital motion of Europa to be Keplerian and Europa's spin axis normal to the orbital plane (obliquity equal to zero). Since Europa's orbit around Jupiter is eccentric (eccentricity $e=0.0094$, see Ta-
Table 1

Numerical values for Europa

\begin{tabular}{lll}
\hline Quantity & Symbol & Value \\
\hline Mass & $M_{E}$ & $4.7998 \times 10^{22} \mathrm{~kg}$ \\
Radius & $R$ & $1560.8 \mathrm{~km}$ \\
Mean motion & $n$ & $1.7691 \mathrm{~d}^{-1}$ \\
Eccentricity & $e$ & 0.0094 \\
Gravitational coefficient & $C_{22}$ & $131.5 \pm 2.5 \times 10^{-6}$ \\
Mean moment of inertia & $I / M_{E} R^{2}$ & $0.3477 \pm 0.0027$ \\
\hline
\end{tabular}

References: JPL Solar System Dynamics (http://ssd.jpl.nasa.gov), Anderson et al. (1998) for $C_{22}$, and this paper for $I$.

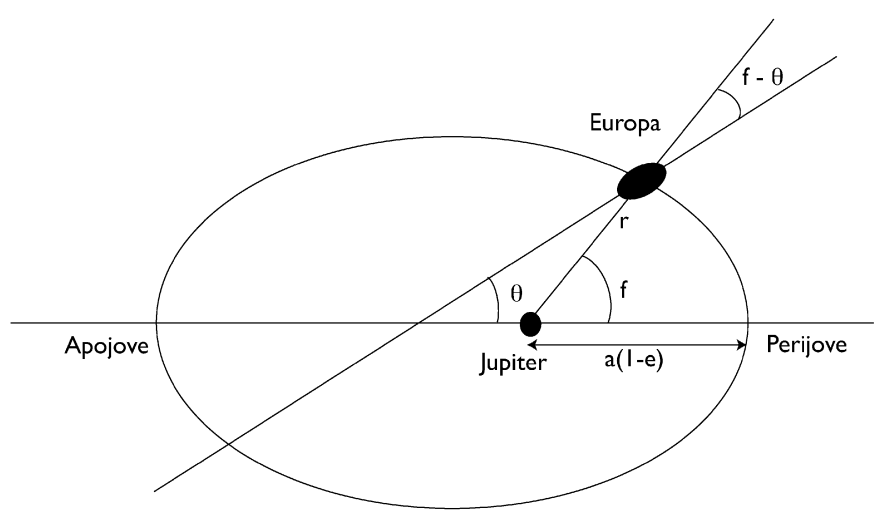

Fig. 1. Geometry of Europa's libration. The long axis of Europa makes an angle $\theta$ with the major axis of the orbit and $f$ is the true anomaly.

ble 1), Europa's orbital speed is variable and Europa does not always show exactly the same face to its central planet. As a consequence, Jupiter exerts a time-variable gravitational torque on Europa that tends to modify the satellite's rotation rate. The corresponding changes in the rotation angle are called longitudinal librations.

Librations can most easily be calculated for a satellite that responds rigidly to an external gravitational force (e.g., Comstock and Bills, 2003). In this case, the angular momentum $H$ of Europa can be written as $H=C \dot{\theta}$, where $C$ is the largest (polar) principal moment of inertia and $\theta$ the angle of rotation between the long axis of Europa and the major axis of the orbit (see Fig. 1). Conservation of angular momentum then shows that the librations are governed by (e.g., Goldreich and Peale, 1966)

$C \ddot{\theta}+\frac{3}{2} n^{2}(B-A)\left(\frac{a}{r}\right)^{3} \sin 2(\theta-f)=0$.

Here, $A<B$ are the two equatorial principal moments of inertia of Europa, $n$ the mean motion of Europa, $a$ the semi-major axis, $f$ the true anomaly, and $r$ the distance between the mass centers of Jupiter and Europa (see Fig. 1). The second term in Eq. (1) represents the opposite of the gravitational torque of Jupiter on Europa.

The rigid rotational response is a good approximation for an entirely solid Europa without internal ocean. The solidity ensures that there will be no relative rotation of the different internal layers and the elastic deformation effect is small. Deformation influences libration by changing the polar moment of inertia of Europa and hence also the angular momentum 
$H=(C+\delta C(t)) \dot{\theta}$, where $\delta C(t)$ is the time-variable part of the polar moment of inertia. The deformation is itself a result of the libration since the changes in the rotation rate lead to a changing centrifugal potential and therefore to a time-variable polar flattening. The associated time-variable part of the polar moment of inertia can be expressed as (see, e.g., Moritz and Mueller, 1987)

$\delta C=k_{2} \frac{4 R^{5} \omega}{9 G}(\dot{\theta}-\omega)$,

where $G$ is the gravitational constant and $R$ the mean radius, $\omega$ the mean rotation rate, and $k_{2}$ the degree-two potential Love number of Europa. The effect of deformation can be included by adding a term

$\omega \frac{\mathrm{d} \delta C}{\mathrm{~d} t}=k_{2} \frac{4 R^{5} \omega^{2}}{9 G} \ddot{\theta}$

to the left-hand member of Eq. (1). This term is more than a factor 1000 smaller than the main term $C \ddot{\theta}$ in Eq. (1), even with the large value of $k_{2}=0.26$ for a model with a subsurface ocean (e.g., Wahr et al., 2006), and will therefore be neglected in this study. We here also neglect the effect of zonal tides on rotation. Zonal tides change the polar flattening of Europa and therefore also the polar moment of inertia. In Section 5, we show that the relative effect of tides on the libration of Europa could be a few percent.

Equation (1) leads to two distinct periodic components for the librations: (i) a libration with natural frequency $\omega_{f}$ and arbitrary amplitude, and (ii) forced librations. By averaging Eq. (1) over an orbital period, the free frequency $\omega_{f}$ can be obtained as (e.g., Comstock and Bills, 2003)

$\omega_{f}=n \sqrt{\frac{3(B-A)}{C}}$.

It is much smaller than the mean motion $n$ since $B-A$ is much smaller than $C$ (the ratio $(B-A) / C$ is of the order of $C_{22}=131.5 \pm 2.5 \times 10^{-6}$, which has been determined from Galileo flybys, Anderson et al., 1998). Dissipation processes due to tides and libration damp the long-periodic free libration, and we will assume its amplitude to be (almost) zero.

The periods of the forced librations are equal to the periods of the Jupiter torque. The main libration term has a period of 3.55 days (the orbital period) and is therefore short-periodic with respect to the free libration. We do not consider the much smaller forced librations with sub-harmonic periods here. Our main goal is to study whether the amplitude of the forced libration at 3.55 days can be used to determine properties of the interior of Europa. Below, without explicit mention, libration refers to the forced libration in longitude of 3.55 days. For small librations, the amplitude of the forced libration can be approximated up to first order in eccentricity $e$ by (e.g., Murray and Dermott, 2000)

$A_{\theta}=-\frac{2 \omega_{f}^{2} e}{n^{2}-\omega_{f}^{2}}=-6 \frac{(B-A)}{C} \frac{n^{2} e}{n^{2}-\omega_{f}^{2}}$.

The principal moments of inertia are the only interior structure parameters involved in the amplitude of the rigid librations of
Europa, and Eq. (5) shows that the libration amplitude is proportional to $(B-A) / C$. The proportionality to the moment of inertia difference $B-A$ stems from the proportionality of the gravitational torque to that difference. Since $C$ is the inertia for rotational motion, the librations are inversely proportional to the polar moment of inertia. The principal moments of inertia of Europa will be determined in Section 3.

\subsection{Shell librations}

\subsubsection{Decoupled shell}

In the case that Europa has a subsurface ocean beneath an icy shell, the solid interior, ocean, and outer shell librate differentially, and the surface libration corresponds to the libration of the icy shell. If it can be assumed that Europa's interior is spherically symmetric, that the ocean is inviscid, and that there is no electromagnetic field, the icy shell would be rotationally decoupled from the deeper interior of Europa. Under these hypotheses, only the shell librates, and the libration can be expressed as Eq. (1) for a rigid Europa by replacing the moments of inertia $A, B$, and $C$ by the shell moments of inertia $A_{s}, B_{s}$, and $C_{s}$ :

$C_{s} \ddot{\theta}_{s}+\frac{3}{2}\left(B_{s}-A_{s}\right) n^{2}\left(\frac{a}{r}\right)^{3} \sin 2\left(\theta_{s}-f\right)=0$.

Here, the angle $\theta_{s}$ is the angle of rotation of the icy shell. As the interior is assumed to be spherically symmetric, it does not contribute to the moment of inertia difference, and the equatorial shell moment of inertia difference is equal to the total Europa $B-A$. As a consequence, the libration amplitude for a decoupled shell is given by

$A_{\theta_{s}}=-\frac{2 \omega_{f s}^{2} e}{n^{2}-\omega_{f s}^{2}}=-6 \frac{(B-A)}{C_{s}} \frac{n^{2} e}{n^{2}-\omega_{f s}^{2}}$,

where the free shell libration frequency $\omega_{f s}$ is given by

$\omega_{f s}=n \sqrt{\frac{3(B-A)}{C_{s}}}=\omega_{f} \sqrt{\frac{C}{C_{s}}}$.

Equation (7) shows that, far from resonance with the free libration, the libration amplitude for the decoupled shell is a factor $C / C_{S}$ larger than the libration amplitude of the corresponding solid model. The free period is a factor $\sqrt{C_{S} / C}$ shorter than the free period of a completely solid Europa, and can be close to the period of the forced libration.

\subsubsection{Gravitational coupling}

More generally, the librations of the different layers are coupled through gravitational, viscous, and electromagnetic forces, and also through the effect of ocean fluid pressure on the nonspherical surfaces of the ocean. Effects of couplings between different layers on rotation variations have been studied extensively for terrestrial planets. For the study of librations of Europa, the best comparison is with the librations of Mercury. The main effect of pressure and gravitational coupling between the solid mantle and an ellipsoidal liquid (outer) core of Mercury is that the mantle libration is larger by the ratio $C / C^{m}$, where 
$C^{m}$ is the polar moment of inertia of the mantle, and the core does not follow the librations of the mantle (e.g., Van Hoolst, 2007). Deviations due to other core-mantle couplings, including viscous coupling, electromagnetic coupling, and gravitational coupling between a solid inner core and the mantle, are below $1 \%$ of the libration signal (Peale et al., 2002; Rambaux et al., 2007). These findings suggest that the libration of the icy shell can be assumed to be rotationally decoupled from the deeper interior of the satellite. However, a main difference with respect to rotation variations of terrestrial planets is that the outer solid layer has a small moment of inertia compared to the moment of inertia of the whole body, in contrast to the mantle and crust of terrestrial planets. Furthermore, the solid interior contributes most to the moment of inertia, also in strong contrast to the terrestrial planets, for which the solid inner core, if it exists, has a much smaller moment of inertia than the moment of inertia of the whole planet. As an example, for the Earth, the moment of inertia of the solid inner core is less than one thousandth of the moment of inertia of the Earth. The solid interior of Europa is therefore expected to have a much larger influence on the rotation variations of the surface for Europa than a solid inner core has on the rotation variations of terrestrial planets. Therefore, we include the direct gravitational coupling between the solid interior and outer icy shell in our study of the librations of Europa.

Gravitational coupling between internal layers of Europa arises when the principal axes of the icy shell and the solid interior below the ocean are not aligned. For example, when the shell rotates out of alignment with the solid interior, it exerts a torque on the solid interior. Since the ocean fills the cavity between the interior and the shell, the mass distribution at the top of the ocean with respect to the solid interior then also changes, and the ocean also exerts a torque on the solid interior. Szeto and $\mathrm{Xu}$ (1997) derived an expression for the gravitational torque exerted by the outer core and mantle of the Earth on the solid inner core:

$$
\begin{aligned}
\Gamma_{z}= & \frac{4 \pi G}{5}\left[\int_{r_{0}^{\mathrm{ICB}}}^{R_{\oplus}} \rho\left(r_{0}^{\prime}\right) \frac{\partial \beta\left(r_{0}^{\prime}\right)}{\partial r_{0}^{\prime}} \mathrm{d} r_{0}^{\prime}\right]\left(B_{\mathrm{IC}}-A_{\mathrm{IC}}\right) \\
& \times \sin 2\left(\theta_{M}-\theta_{\mathrm{IC}}\right)
\end{aligned}
$$

(see also Xu et al., 2000). Here, $R_{\oplus}$ is the mean radius of the Earth, $\theta$ the rotation angle, $r_{0}$ the mean radius of an ellipsoidal internal surface, and $\beta$ the equatorial flattening

$\beta=\frac{a\left(r_{0}\right)-b\left(r_{0}\right)}{a\left(r_{0}\right)}$,

where $a\left(r_{0}\right)$ and $b\left(r_{0}\right)$ are the two equatorial radii of that surface. Subscripts $M$ and IC are used to denote the mantle and inner core, respectively, and ICB refers to the inner core boundary. Expression (9) is valid for the gravitational torque of an ellipsoidal shell on an ellipsoidal volume that is internal to the shell and we will use it to determine the gravitational coupling in Europa. The integral between brackets in Eq. (9) is over the mean radial coordinate $r_{0}$ of the outer shell, and the equatorial moment of inertia difference is of the inner volume. If either of

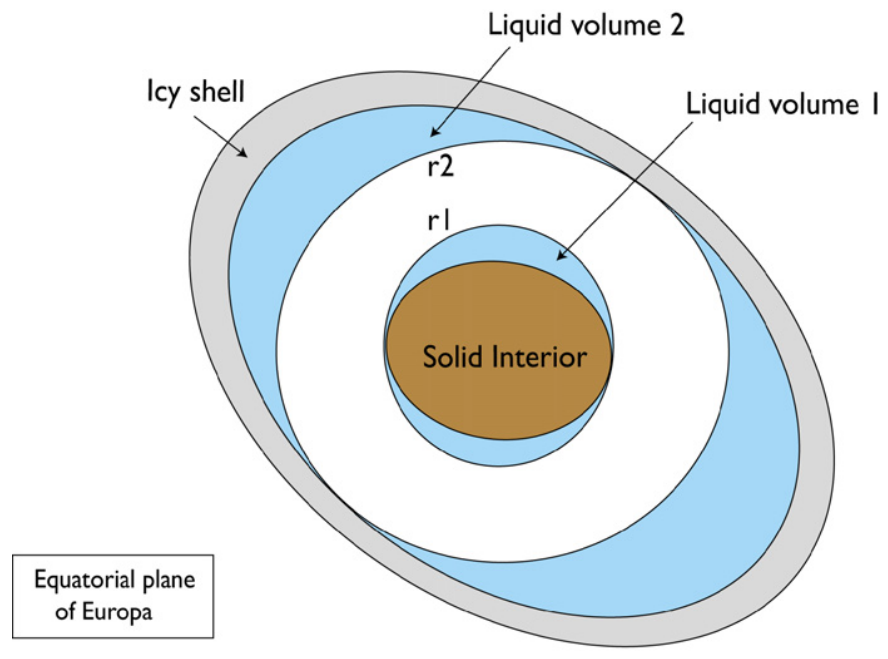

Fig. 2. Different internal regions in Europa for the study of internal gravitational coupling (not to scale).

the volumes is spherically symmetric, the torque will be zero since then either the integral over the flattening of the outer volume or the moment of inertia difference of the inner volume will be zero.

For ease of calculation and interpretation of the coupling, we consider two spheres inside the ocean of Europa, which we assume to be homogeneous in density. Sphere 1 with radius $r_{1}$ encloses the solid interior and sphere 2 with radius $r_{2}$ is the largest sphere in the ocean beneath the shell (see Fig. 2). The liquid in the volume between spheres 1 and 2 does not contribute to gravitational coupling with the shell or with the interior since its mass distribution with respect to the two solid regions does not change when the solids rotate. Therefore, we only need to consider the gravitational coupling between the regions interior and exterior to the spherical volume between spheres 1 and 2. Region 1 comprises the solid interior and volume 1 between sphere 1 and the solid interior, and region 2 covers the shell and volume 2 between the shell and sphere 2 (see Fig. 2). We note that there is also no pressure coupling of the liquid in the volume between spheres 1 and 2 with the mass in regions 1 and 2 since the interfaces are spherical. The integral in the gravitational torque Eq. (9) must then be evaluated over region 2 (the shell and volume 2) and the moment of inertia difference in Eq. (9) applies to region 1 (the solid interior plus volume 1). The integral can be decomposed as

$$
\begin{aligned}
\int_{r_{2}}^{R} \rho\left(r_{0}^{\prime}\right) \frac{\partial \beta\left(r_{0}^{\prime}\right)}{\partial r_{0}^{\prime}} \mathrm{d} r_{0}^{\prime}= & \int_{\text {volume } 2} \rho\left(r_{0}^{\prime}\right) \frac{\partial \beta\left(r_{0}^{\prime}\right)}{\partial r_{0}^{\prime}} \mathrm{d} r_{0}^{\prime} \\
& +\int_{\text {shell }} \rho\left(r_{0}^{\prime}\right) \frac{\partial \beta\left(r_{0}^{\prime}\right)}{\partial r_{0}^{\prime}} \mathrm{d} r_{0}^{\prime},
\end{aligned}
$$

where $R$ is the mean radius of Europa. We assume that the icy shell is of constant density. Since also the ocean is assumed to be homogeneous in density, the integrals can be expressed in terms of the equatorial flattenings at the top and bottom of the 
layers considered. We then have

$\int_{r_{2}}^{R} \rho\left(r_{0}^{\prime}\right) \frac{\partial \beta\left(r_{0}^{\prime}\right)}{\partial r_{0}^{\prime}} \mathrm{d} r_{0}^{\prime}=\left(\rho_{o}-\rho_{s}\right) \beta_{o}+\rho_{s} \beta_{s}$,

where $\rho_{o}$ and $\rho_{s}$ are the densities of the ocean and the shell, respectively, and $\beta_{o}$ and $\beta_{s}$ their respective equatorial flattenings.

The moment of inertia difference of region 1 can be decomposed as

$$
\begin{aligned}
B-A & =\left(B_{i}-A_{i}\right)+\left(B_{1}-A_{1}\right) \\
& =\frac{8 \pi}{15} \int_{0}^{r_{i}} \rho\left(r_{0}^{\prime}\right) \frac{\mathrm{d}\left(\beta r_{0}^{\prime 5}\right)}{\mathrm{d} r_{0}^{\prime}} \mathrm{d} r_{0}^{\prime}+\frac{8 \pi}{15} \int_{r_{i}}^{r_{1}} \rho\left(r_{0}^{\prime}\right) \frac{\mathrm{d}\left(\beta r_{0}^{\prime 5}\right)}{\mathrm{d} r_{0}^{\prime}} \mathrm{d} r_{0}^{\prime},
\end{aligned}
$$

where subscript $i$ denotes the solid interior (mantle and core). The moment of inertia difference of volume 1 ,

$B_{1}-A_{1}=-\frac{8 \pi}{15} \rho_{o} \beta_{i} r_{i}^{5}$,

is equal to the opposite of the moment of inertia difference that the solid interior volume would have if it had a density $\rho_{o}$. The deep interior of Europa is thought to be composed of a silicate mantle and an iron rich core (Anderson et al., 1998). Although the iron core could be (partially) liquid, we assume that the rotation of the core is locked to the rotation of the mantle as would be the case for a solid core. By assuming the core and mantle to be homogeneous in density, the equatorial moment of inertia difference of the interior can be written as

$B_{i}-A_{i}=\frac{8 \pi}{15}\left[\rho_{m} \beta_{i} r_{i}^{5}+\left(\rho_{c}-\rho_{m}\right) \beta_{c} r_{c}^{5}\right]$,

where $\rho_{c}$ and $\rho_{m}$ are the densities (considered constant) of the core and mantle, respectively, and $\beta_{c}$ and $r_{c}$ are the equatorial flattening and radius of the core. By substituting Eqs. (12), (14), and (15) into Eq. (9) the gravitational torque of the outer region 2 on the inner region 1 can be expressed as

$$
\begin{aligned}
\Gamma_{z}= & \frac{4 \pi G}{5} \frac{8 \pi}{15}\left[\rho_{s} \beta_{s}+\left(\rho_{o}-\rho_{s}\right) \beta_{o}\right] \\
& \times\left[\left(\rho_{m}-\rho_{o}\right) \beta_{i} r_{i}^{5}+\left(\rho_{c}-\rho_{m}\right) \beta_{c} r_{c}^{5}\right] \sin 2\left(\theta_{s}-\theta_{i}\right) \\
= & K \sin 2\left(\theta_{s}-\theta_{i}\right) .
\end{aligned}
$$

The gravitational coupling strength $K$ depends on the flattening and density of the icy shell and solid interior. If the solid interior could be assumed to have a constant density $\rho_{i}$, we would obtain (from Eqs. (13), (14), and (15))

$B-A=\left(1-\frac{\rho_{o}}{\rho_{i}}\right)\left(B_{i}-A_{i}\right)$,

and the gravitational torque on the interior would simplify to

$$
\begin{aligned}
\Gamma_{z}= & \frac{4 \pi G}{5}\left[\rho_{s} \beta_{s}+\left(\rho_{o}-\rho_{s}\right) \beta_{o}\right]\left(1-\frac{\rho_{o}}{\rho_{i}}\right) \\
& \times\left(B_{i}-A_{i}\right) \sin 2\left(\theta_{s}-\theta_{i}\right) .
\end{aligned}
$$

The factor $\left(1-\rho_{o} / \rho_{i}\right)$ shows that the gravitational torque is zero when the liquid and the solid interior have equal density. In that case, a change in orientation would not lead to a change in

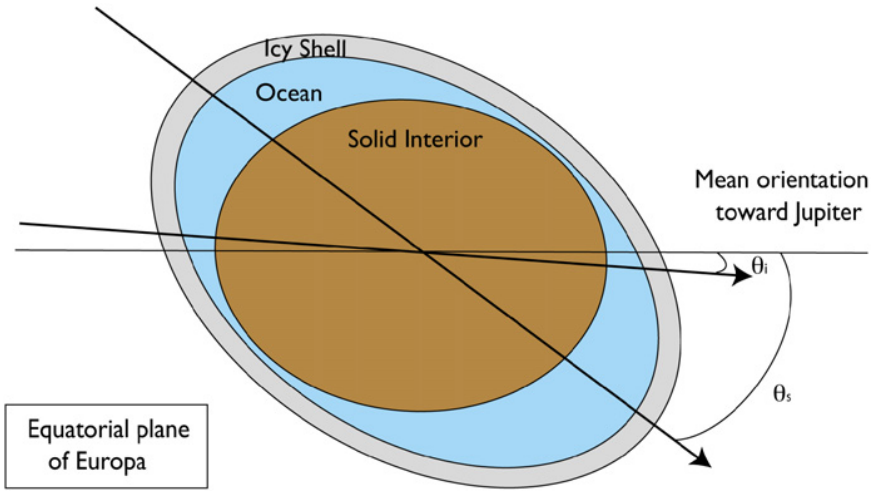

Fig. 3. Geometry of Europa's shell and interior libration (not to scale).

the gravitational potential and there would not be a gravitational torque. A similar expression as Eq. (18) for the gravitational torque on the solid inner core of terrestrial planets has been used by, e.g., Xu et al. (2000) and Peale et al. (2002).

We next write angular momentum equations for the internal layers of Europa. We neglect the dynamics of the ocean and keep only angular momentum equations for the icy shell and the solid interior. However, the torque expression (16) is calculated between the solid regions with inclusion of the liquid volumes 1 and 2 . We therefore consider the angular momentum equations for the solid regions including the surrounding liquid volumes, which have been called regions 1 and 2 above. However, since the volumes 1 and 2 are very thin (of the order of $r_{1} \beta\left(r_{1}\right)$ and $r_{2} \beta\left(r_{2}\right)$, respectively), their polar moments of inertia are a factor $\beta$ smaller than the moments of inertia of the corresponding solid regions. Therefore, correct up to the lowest order in the equatorial flattening, the change in angular momentum of the solid interior and volume 1 can be set equal to the change in angular momentum of the solid interior, or, alternatively, Eq. (16) can be considered as the gravitational torque of the icy shell on the solid interior in the angular momentum equation for the solid interior. Similarly, it is the opposite of the torque on the icy shell in the angular momentum equation for the shell. The libration of Europa can then be described by

$$
\begin{aligned}
& C_{s} \ddot{\theta}_{s}+\frac{3}{2}\left(B_{s}-A_{s}\right) n^{2}\left(\frac{a}{r}\right)^{3} \sin 2\left(\theta_{s}-f\right) \\
& =-K \sin 2\left(\theta_{s}-\theta_{i}\right), \\
& C_{i} \ddot{\theta}_{i}+\frac{3}{2}\left(B_{i}-A_{i}\right) n^{2}\left(\frac{a}{r}\right)^{3} \sin 2\left(\theta_{i}-f\right) \\
& =K \sin 2\left(\theta_{s}-\theta_{i}\right),
\end{aligned}
$$

where the subscripts $s$ and $i$ are for shell and interior, respectively (see Fig. 3).

\subsubsection{Shell and interior librations}

To solve Eqs. (19) and (20), we introduce the small libration angles $\gamma_{s}=\theta_{s}-M$ and $\gamma_{i}=\theta_{i}-M$ and expand the equations as a series in mean anomaly $M$ and eccentricity $e$ by using wellknown expansions for $(a / r)^{3}, \cos f$, and $\sin f$ (Cayley, 1861). 
We then have

$$
\begin{aligned}
C_{s} & \ddot{\gamma}_{s} \\
+ & \frac{3}{2}\left(B_{s}-A_{s}\right) n^{2} \sum_{k=-\infty}^{+\infty} X_{-k}^{-3,2}(e) \sin \left[2 \gamma_{s}+(2+k) M\right] \\
& =-2 K\left(\gamma_{s}-\gamma_{i}\right), \\
C_{i} \ddot{\gamma}_{i} & +\frac{3}{2}\left(B_{i}-A_{i}\right) n^{2} \sum_{k=-\infty}^{+\infty} X_{-k}^{-3,2}(e) \sin \left[2 \gamma_{i}+(2+k) M\right] \\
& =2 K\left(\gamma_{s}-\gamma_{i}\right),
\end{aligned}
$$

where the eccentricity functions $X_{-k}^{-3,2}(e)$ are Hansen coefficients (Hansen, 1855; Hughes, 1981). Next, we linearize the equations in eccentricity and libration angles $\gamma_{s}$ and $\gamma_{i}$. Since the Hansen coefficients $X_{k}^{n, m}$ are of order $e^{|k-m|}$ in eccentricity (e.g., Plummer, 1918), only Hansen coefficients $X_{-k}^{-3,2}(e)$ in Eqs. (21) and (22) with index $k$ equal to $-1,-2$, and -3 have to be retained. These are $X_{1}^{-3,2}(e)=-e / 2, X_{2}^{-3,2}(e)=1$, $X_{3}^{-3,2}(e)=7 e / 2$, up to first order in eccentricity, and we then have

$C_{s} \ddot{\gamma}_{s}+\sigma_{s}^{2} C_{s} \gamma_{s}+2 K\left(\gamma_{s}-\gamma_{i}\right)=2 e \sigma_{s}^{2} C_{s} \sin M$,

$C_{i} \ddot{\gamma}_{i}+\sigma_{i}^{2} C_{i} \gamma_{i}-2 K\left(\gamma_{s}-\gamma_{i}\right)=2 e \sigma_{i}^{2} C_{i} \sin M$,

where $\sigma_{s}$ and $\sigma_{i}$ are defined as

$\sigma_{s}=n \sqrt{\frac{3\left(B_{s}-A_{s}\right)}{C_{s}}}$,

$\sigma_{i}=n \sqrt{\frac{3\left(B_{i}-A_{i}\right)}{C_{i}}}$.

We assume that the librations are zero at perijove and set $\gamma_{s}=A_{\theta}^{s} \sin M$ and $\gamma_{i}=A_{\theta}^{i} \sin M$ where $A_{\theta}^{s}$ and $A_{\theta}^{i}$ are the amplitudes of the libration of 3.55 days for the shell and the interior, respectively. By substituting these expressions in Eqs. (23) and (24), we obtain a system of two algebraic equations in $A_{\theta}^{s}$ and $A_{\theta}^{i}$, which we solve by using Kramer's method:

$A_{\theta}^{s}=\frac{\phi_{s_{1}}}{n^{2}-\omega_{f 1}^{2}}+\frac{\phi_{S_{2}}}{n^{2}-\omega_{f 2}^{2}}$,
$A_{\theta}^{i}=\frac{\phi_{i_{1}}}{n^{2}-\omega_{f 1}^{2}}+\frac{\phi_{i_{2}}}{n^{2}-\omega_{f 2}^{2}}$,

where the coefficients in the numerators are given by

$$
\begin{aligned}
\phi_{s_{1}}= & 2 e\left[\sigma_{s}^{2}\left(\omega_{f 1}^{2}-\sigma_{i}^{2}-2 \frac{K}{C_{i}}\right)-2 \sigma_{i}^{2} \frac{K}{C_{s}}\right] \\
& \times n^{-2}\left(\omega_{f 2}^{2}-\omega_{f 1}^{2}\right)^{-1}, \\
\phi_{s_{2}}= & 2 e\left[\sigma_{s}^{2}\left(\sigma_{i}^{2}-\omega_{f 2}^{2}+2 \frac{K}{C_{i}}\right)+2 \sigma_{i}^{2} \frac{K}{C_{s}}\right] \\
& \times n^{-2}\left(\omega_{f 2}^{2}-\omega_{f 1}^{2}\right)^{-1}, \\
\phi_{i_{1}}= & 2 e\left[\sigma_{i}^{2}\left(\sigma_{s}^{2}-\omega_{f 2}^{2}+2 \frac{K}{C_{s}}\right)+2 \sigma_{s}^{2} \frac{K}{C_{i}}\right] \\
& \times n^{-2}\left(\omega_{f 2}^{2}-\omega_{f 1}^{2}\right)^{-1},
\end{aligned}
$$

$$
\begin{aligned}
\phi_{i_{2}}= & 2 e\left[\sigma_{i}^{2}\left(\omega_{f 1}^{2}-\sigma_{s}^{2}-2 \frac{K}{C_{s}}\right)-2 \sigma_{s}^{2} \frac{K}{C_{i}}\right] \\
& \times n^{-2}\left(\omega_{f 2}^{2}-\omega_{f 1}^{2}\right)^{-1} .
\end{aligned}
$$

The eigenfrequencies $\omega_{f 1}$ and $\omega_{f 2}$ are the frequencies of the two free librations, which can be obtained by averaging Eqs. (23) and (24) over one orbital cycle and assuming that the libration angles vary slowly. We have two free frequencies because the librations of two coupled layers are considered. By taking the time-dependence of the libration amplitudes as $\exp (\mathrm{i} \sigma t)$, we obtain a system of two homogeneous algebraic equations, which has non-zero solutions for the two free frequencies given by

$\omega_{f 1}^{2}=\frac{1}{2}\left(\sigma_{i}^{2}+v^{2}+\sigma_{s}^{2}+\sqrt{\Delta}\right)$,

$\omega_{f 2}^{2}=\frac{1}{2}\left(\sigma_{i}^{2}+v^{2}+\sigma_{s}^{2}-\sqrt{\Delta}\right)$,

where

$$
\begin{aligned}
\Delta= & \sigma_{i}^{4}+\sigma_{s}^{4}+v^{4}+2 \sigma_{i}^{2} v^{2}+2 v^{2} \sigma_{s}^{2}-2 \sigma_{i}^{2} \sigma_{s}^{2}-8 \sigma_{i}^{2} K / C_{s} \\
& -8 \sigma_{s}^{2} K / C_{i},
\end{aligned}
$$

and

$v=\sqrt{2 K \frac{C_{s}+C_{i}}{C_{S} C_{i}}}$

is the oscillation frequency of the differential rotation between shell and interior when the external gravitational coupling of Jupiter is neglected [see also Buffett (1996) and Xu et al. (2000) for the case of the Earth].

\section{Interior structure models}

\subsection{Introduction}

If Europa reacts rigidly to the gravitational torque of Jupiter, $B-A$ and $C$ are the only interior structure quantities necessary for the calculation of the librations. When differential libration of the internal layers is considered, the polar moments of inertia and the equatorial flattenings or equatorial moment of inertia differences of the external icy shell as well as the internal layers need to be known to characterize the librational response. To be able to study the effect of the interior on libration, we construct models of the internal structure of Europa and calculate the key interior parameters for libration.

We use a three-step procedure. First, from the observed $C_{22}$ gravity coefficient, we determine the mean moment of inertia of Europa. To that end, we reconsider and extend the classical Radau equation, which relates the polar flattening to the mean moment of inertia $I=(A+B+C) / 3$, to synchronously rotating satellites flattened by rotation and permanent tides (Section 3.2). Secondly, we construct spherically symmetric models of the internal structure of Europa that satisfy the observed mass $M_{E}=4.7998 \times 10^{22} \mathrm{~kg}$, radius $R=1560.8 \mathrm{~km}$ (see Table 1) and the estimated mean moment of inertia (Section 3.3). Finally, we apply Clairaut theory to calculate the polar and equatorial flattening as a function of radial distance to the center of 
Europa, and determine the polar principal moments of inertia and the equatorial moment of inertia differences for the internal layers of our Europa models (Section 3.3).

\subsection{Equilibrium figure of Europa}

Rotation flattens Europa at the poles and creates an equatorial bulge, whereas the tides stretch Europa in the direction to Jupiter and cause a contraction in the direction perpendicular to Jupiter. As a result, the longest axis is expected to be along the planet-satellite line at perijove and the short axis is parallel to the rotation axis. The deformations depend on the perturbing potentials as well as on the response of Europa to the forcing. The centrifugal potential $U_{r}$ due to rotation can be expressed as

$U_{r}(\vec{r})=\frac{1}{3} \omega^{2} r^{2}\left[1-P_{2}^{0}(\cos \varphi)\right]$,

and the static degree-two tidal potential $U_{t}$ responsible for the permanent tidal perturbation as

$U_{t}(\vec{r})=-\frac{1}{2} \frac{G M_{J}}{a}\left(\frac{r}{a}\right)^{2}\left[P_{2}^{0}(\cos \varphi)-\frac{1}{2} P_{2}^{2}(\cos \varphi) \cos 2 \lambda\right]$.

Here, $\omega$ is the mean rotation rate of Europa $\left(2 \pi / 3.55\right.$ days $\left.^{-1}\right)$, $M_{J}$ the mass of Jupiter, $a$ the semi-major axis of Europa's orbit, $r$ the radial distance from the mass center of Europa to the point considered with position vector $\vec{r}$, and $\varphi$ and $\lambda$ are co-latitude and longitude of $\vec{r}$. The geodetic sign convention $\vec{F}=\vec{\nabla} U$ is used. Since the perturbing potential can be expressed in terms of the associated Legendre functions $P_{2}^{0}(\cos \varphi)$ and $P_{2}^{2}(\cos \varphi)$, the perturbed body, assumed to be in hydrostatic equilibrium, will take the form of a triaxial ellipsoid. Any surface of constant density can then be expressed by

$r=r_{0}\left[1-\frac{2}{3} \alpha\left(r_{0}\right) P_{2}^{0}(\cos \varphi)+\frac{1}{6} \beta\left(r_{0}\right) P_{2}^{2}(\cos \varphi) \cos 2 \lambda\right]$,

where $r_{0}$ is the mean radius of the ellipsoidal surface, $\alpha=[(a+$ b) $/ 2-c] /[(a+b) / 2]$ is the polar flattening, and $\beta=(a-b) / a$ is the equatorial flattening (see, e.g., Van Hoolst and Dehant, 2002). We use radii of the three principal axes of the ellipsoids $a\left(r_{0}\right)>b\left(r_{0}\right)>c\left(r_{0}\right)$.

The rotational deformation is described by Clairaut's differential equation

$\frac{\mathrm{d}^{2} \alpha}{\mathrm{d} r_{0}^{2}}+\frac{6}{r_{0}} \frac{\rho}{\bar{\rho}} \frac{\mathrm{d} \alpha}{\mathrm{d} r_{0}}-\frac{6}{r_{0}^{2}}\left(1-\frac{\rho}{\bar{\rho}}\right) \alpha=0$,

and an associated boundary condition (see Eq. (40) below, e.g., Jeffreys, 1952). Here, $\bar{\rho}$ is the mean density in a sphere of radius $r_{0}$. The mean moment of inertia $I$ can be determined from the polar flattening $\alpha$ by using Radau's (1885) accurate approximation to Clairaut's equation as

$\frac{I}{M_{E} R^{2}}=\frac{2}{3}\left\{1-\frac{2}{5}\left[1+\frac{\mathrm{d} \ln \alpha}{\mathrm{d} \ln r_{0}}(R)\right]^{1 / 2}\right\}$,

where the logarithmic derivative of the polar flattening is evaluated at the surface $r_{0}=R$ (see, e.g., Jeffreys, 1952).
For the calculation of Europa's mean moment of inertia $I$ from the polar flattening using Clairaut theory, we not only have to consider the rotational effect, but also have to include the polar flattening due to the static tides. Therefore, the perturbing degree-two, order-zero potential $U_{p}^{2,0}$ to be considered in Clairaut theory must also include the tidal part:

$U_{p}^{2,0}=U_{r}^{2,0}+U_{t}^{2,0}=-\left[\frac{1}{3} \omega^{2}+\frac{1}{2} \frac{G M_{J}}{a^{3}}\right] r^{2} P_{2}^{0}(\cos \varphi)$.

We see that both perturbing potentials depend on $r^{2}$. For a synchronously rotating satellite, $M_{J} / a^{3}=\omega^{2} / G$, and the expression for $U_{p}^{2,0}$ simplifies to

$U_{p}^{2,0}=-\frac{5}{6} \omega^{2} r^{2} P_{2}^{0}(\cos \varphi)=\frac{5}{2} U_{r}^{2,0}$.

The perturbing potential including both the centrifugal and the degree-two, order-zero static tidal potential is 5/2 times larger than the centrifugal potential. Since the perturbing potential has the same spatial dependence as the centrifugal potential and differs from it only by a constant factor, the calculation of the deformation of Europa including static tides is similar to that for rotation, and Clairaut's theory can easily be extended to this more general case.

As can be seen from Eq. (36) Clairaut's equation does not contain the perturbing potential, and remains unaltered when the tidal potential is included. Similarly, Eq. (37) for the moment of inertia remains valid. The perturbing potential enters the problem through the boundary condition of Clairaut's equation, which does depend on the perturbing potential. For rotational deformation only, $\alpha_{r}$, we have at the surface (see, e.g., Moritz, 1990)

$\frac{\mathrm{d} \alpha_{r}}{\mathrm{~d} r_{0}}(R)=\frac{1}{R}\left[\frac{5}{2} q-2 \alpha_{r}(R)\right]$,

where $q$ is the ratio of the centrifugal acceleration to the gravitational acceleration $q=\left(\omega^{2} R^{3}\right) /\left(G M_{E}\right)$ and the polar flattening and its derivative with respect to $r_{0}$ are evaluated at the surface $r_{0}=R$. This equation can readily be transformed to include the tidal effect on the polar flattening by multiplying the term in $q$ stemming from the perturbing potential by $5 / 2$ (see Eq. (39)). We then have the following expressions for the polar and equatorial flattening at the surface

$\frac{\mathrm{d} \alpha}{\mathrm{d} r_{0}}(R)=\frac{1}{R}\left[\frac{25}{4} q-2 \alpha(R)\right]$.

By substituting Eq. (41) into Eq. (37), the derivative of the flattening in Eq. (37) can be expressed in terms of the flattening itself. We then have

$\frac{I}{M_{E} R^{2}}=\frac{2}{3}\left\{1-\frac{2}{5}\left[\frac{25}{4} \frac{q}{\alpha(R)}-1\right]^{1 / 2}\right\}$,

which is the extension of the Radau equation (Radau, 1885; see also Jeffreys, 1952; Moritz, 1990; Hubbard, 1984) to a synchronously rotating satellite that is deformed by rotation and tides.

The surface polar flattening $\alpha(R)$ can be expressed in terms of the observed $J_{2}$ gravity coefficient by assuming that the outer 
surface is an equipotential surface (consistent with our assumption of hydrostatic equilibrium). We write the total potential as the sum of the gravitational potential of Europa, the centrifugal potential, and the static tidal potential:

$$
\begin{aligned}
U= & \frac{G M_{E}}{r}\left[1-J_{2} P_{2}^{0}(\cos \varphi)+C_{22} P_{2}^{2}(\cos \varphi) \cos 2 \lambda\right] \\
& +U_{r}+U_{t} .
\end{aligned}
$$

By substituting Eqs. (33), (34), and (35) into Eq. (43), we have correct up to the first order in the flattenings

$$
\begin{aligned}
U= & \frac{G M_{E}}{R}\left\{1+\frac{1}{3} q+\left[\frac{2}{3} \alpha(R)-J_{2}-\frac{5}{6} q\right] P_{2}^{0}(\cos \varphi)\right. \\
& \left.+\left[C_{22}+\frac{1}{4} q-\frac{1}{6} \beta(R)\right] P_{2}^{2}(\cos \varphi) \cos 2 \lambda\right\} .
\end{aligned}
$$

Since $U$ must be constant on the external equipotential surface, the coefficients of $P_{2}^{0}$ and $P_{2}^{2}$ must be equal to zero. We then have the following expressions for the surface flattenings

$\alpha(R)=\frac{3}{2} J_{2}+\frac{5}{4} q$,

and

$\beta(R)=6 C_{22}+\frac{3}{2} q$.

By substituting Eq. (45) in Eq. (42), the extension of the Radau equation can alternatively be written as

$\frac{I}{M_{E} R^{2}}=\frac{2}{3}\left[1-\frac{2}{5}\left(\frac{25}{4} \frac{q}{\frac{3}{2} J_{2}+\frac{5}{4} q}-1\right)^{1 / 2}\right]$.

By using the observed $C_{22}=131.5 \pm 2.5 \times 10^{-6}$ (from Galileo flybys, Anderson et al., 1998), the hydrostatic relation $J_{2}=$ $(10 / 3) C_{22}$ (see Eqs. (A.1) and (A.2) in Appendix A) and $q=$ $4.9773 \times 10^{-4}$, the mean moment of inertia of Europa can be determined as $I / M_{E} R^{2}=0.3477 \pm 0.0027$, which is our third constraint on the interior structure models of Europa, in addition to $R$ and $M_{E}$.

In the literature, an alternative form of the Radau equation that uses the fluid Love number $k_{f}$ (see Eq. (A.3)) is sometimes used for rotationally and tidally deformed icy satellites, although the relation has, up to our knowledge, only been proved for rotationally deformed bodies. We show in Appendix A that formulation of the Radau equation remains valid for bodies that are also tidally deformed. We also note that in most works, including Jeffreys (1952) and Moritz (1990), the symbol $C$ is used instead of $I$ in Radau's equation, suggesting that the polar moment of inertia is meant. Nevertheless, it is clear from their expression for $C$ that the mean moment of inertia $I$ is used [see also Denis et al. (1998) for a discussion of this common mistake]. For a similar equation adapted to the polar moment of inertia $C$, see Darwin (1899).

\subsection{Spherically symmetric models}

We calculated a large set of spherically symmetric models of the interior structure of Europa. Europa is considered to be differentiated into four layers with homogeneous density:

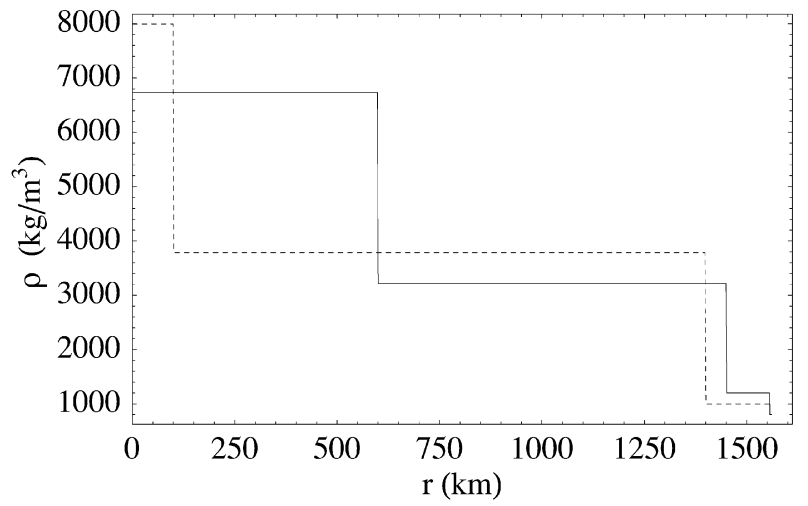

Fig. 4. Density profile for two representative models of the interior structure of Europa. The first model (dashed line) has a small core with a radius of $100 \mathrm{~km}$ and a density of $7995 \mathrm{~kg} \mathrm{~m}^{-3}$. The water layer (ocean + shell) is $160.8 \mathrm{~km}$ thick, and the thickness of the icy shell is $10 \mathrm{~km}$. The second model (solid line) has a much larger (radius $600 \mathrm{~km}$ ) and less dense (density $6735 \mathrm{~kg} \mathrm{~m}^{-3}$ ) core. Also the water layer $(110.8 \mathrm{~km})$ and the icy shell $(5 \mathrm{~km})$ are thinner for the second model.

(1) an outer solid icy shell, (2) an ocean, (3) a rocky mantle, and (4) a metallic core (see Fig. 4). Layers of homogeneous density can be assumed since the layers are likely to be approximately homogeneous in composition and the effect of pressure on density is small for the relatively small satellite (Sohl et al., 2002). Eight parameters then characterize our models: the radii and the densities of the four layers. Since we only have three constraints, we choose values in a prescribed range for five parameters and estimate the three other parameters such that the models satisfy the three constraints on the mass, radius, and mean moment of inertia. The three estimated parameters are the ocean thickness and the densities of the core and the mantle (see Table 2).

We have used the following density ranges for the four layers. For an ocean and icy shell composed of pure water, the densities are $\rho_{o} \approx 1000 \mathrm{~kg} \mathrm{~m}^{-3}$ and $\rho_{s} \approx 920 \mathrm{~kg} \mathrm{~m}^{-3}$, where subscripts $o$ and $s$ are used to indicate the ocean and the shell, respectively. We consider $\rho_{o}$ and $\rho_{s}$ to be between 800 and $1200 \mathrm{~kg} \mathrm{~m}^{-3}$ to include the effects of pressure, temperature, ice porosity, and compositional impurities, such as the salts detected on the surface of Europa (McCord et al., 1998; Kargel et al., 2000). In our models, we only considered density values of 800,1000 and $1200 \mathrm{~kg} \mathrm{~m}^{-3}$ for both the ocean and the shell. The density $\rho_{c}$ of the metallic core varies between $8000 \mathrm{~kg} \mathrm{~m}^{-3}$ (for a pure iron core) and $5150 \mathrm{~kg} \mathrm{~m}^{-3}$ (for an iron-sulfur assemblage of eutectic composition) depending on the amount of light elements such as sulfur (Anderson et al., 1998). The densities of the silicate rocks of the mantle are within the range $3000 \mathrm{~kg} \mathrm{~m}^{-3}<\rho_{m}<4450 \mathrm{~kg} \mathrm{~m}^{-3}$ (Anderson et al., 1998).

The thickness of the icy shell has been estimated in many studies. The lowest estimates mostly come from analyses of regional surface features, whereas impact studies and thermal models give larger thicknesses. The estimates range from hundreds of meters to tens of kilometers (for an overview, see Billings and Kattenhorn, 2005). Here, we consider a broad range of thicknesses between 5 and $100 \mathrm{~km}$. Since topography 
Table 2

Size and density of the four internal layers of the Europa models

\begin{tabular}{ll}
\hline Layer & Thickness $(\mathrm{km}) /$ radius $(\mathrm{km})$ \\
\hline Shell & Thickness: $5,10,20,30,40,50,60,70,80,90,100$ \\
Ocean & Thickness: $10.8-155.8$ \\
Mantle & Radius: $(1300),(1350), 1400,1450,(1500)$ \\
Core & Radius: $100,200,300,400,500,600,700,(800),(1000)$ \\
\hline
\end{tabular}

The ocean thickness and the densities of the core and the mantle are calculated for the given values of the other interior parameters. The input parameters in parentheses do not satisfy the given constraints on mass, radius and moment of inertia.

with a range of a few $\mathrm{km}$ is observed on Europa (Schenk, 2001), it seems likely that the shell thickness is at least a few $\mathrm{km}$, and we here take $5 \mathrm{~km}$ as our lower bound. The upper bound is taken larger than most estimates to include uncertainties in the shell thickness models and smaller than the depth of the combined ice and water layers, which is between 115 and $165 \mathrm{~km}$ for our models, in agreement with Anderson et al. (1998). Density profiles of Europa for two representative models of the in total 277 models are shown in Fig. 4.

\subsection{Internal flattening and principal moments of inertia}

In this section, we calculate the permanent rotational and tidal deformation of our set of spherically symmetric interior structure models of Europa. In particular, we compute the polar moment of inertia and the equatorial moment of inertia difference of the internal layers, which are the quantities needed for our study of the librations of Europa. The equatorial moment of inertia difference of the different layers of Europa can most easily be calculated by applying Clairaut's theory, which is normally only used for the calculation of the polar flattening. Here, instead of considering the $z$-axis along the rotation axis as for the calculation of rotational flattening, we assume it to coincide with the longest principal axis of inertia, which is in the equatorial plane. With this choice of axis, the static tidal potential can be expressed as

$U_{t}=\frac{G M_{J}}{a}\left(\frac{r}{a}\right)^{2} P_{2}^{0}\left(\cos \varphi^{\prime}\right)=\omega^{2} r^{2} P_{2}^{0}\left(\cos \varphi^{\prime}\right)$,

where the angle $\varphi^{\prime}$ is measured from the new z-axis. The potential takes the same form as the centrifugal potential with respect to the rotation axis, except for an additional factor -3 . As a consequence, the equatorial flattening $\beta$ can be derived from Clairaut's equation for $\beta$ and the associated boundary condition

$\frac{\mathrm{d} \beta}{\mathrm{d} r_{0}}(R)=\frac{1}{R}\left[\frac{15}{2} q-2 \beta(R)\right]$,

which follows from the Clairaut boundary condition (40) by taking into account that the perturbing potential (Eq. (48)) is three times larger in absolute values than the centrifugal potential (Eq. (33)). Here, $\beta$ and its derivative with respect to the radial coordinate $r_{0}$ are evaluated at the surface $r_{0}=R$.

Since both Clairaut's equation and its boundary condition are linear in the flattening, the solution of Clairaut's equation for the tidally induced equatorial flattening $\beta\left(r_{0}\right)$ can be derived from the solution of Clairaut's equation for the rotational

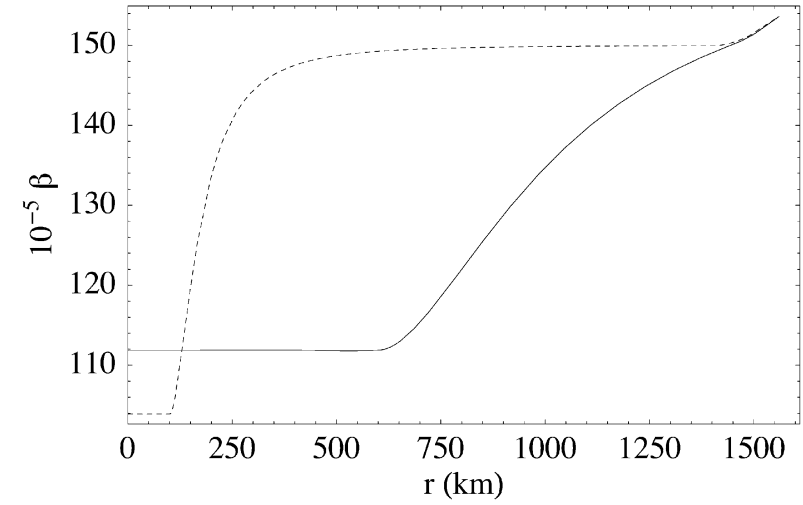

Fig. 5. Internal polar flattening $\beta\left(r_{0}\right)$ for the same models of the interior structure of Europa as in Fig. 4.

polar flattening as $\beta\left(r_{0}\right)=3 \alpha_{r}\left(r_{0}\right)$. Similarly, it follows from a comparison of boundary conditions (40) and (41) that the polar flattening due to rotation and static tides $\alpha\left(r_{0}\right)=\frac{5}{2} \alpha_{r}\left(r_{0}\right)$. For synchronous satellites deformed by rotation and static tides, we then have the relation between polar flattening and equatorial flattening $\alpha\left(r_{0}\right)=\frac{5}{6} \beta\left(r_{0}\right)$, which is valid at any radial distance from the center of the satellite. Internal flattening profiles for two representative models are shown in Fig. 5.

The principal moments of inertia of our models can be determined from $\alpha$ and $\beta$. For a homogeneous model with mean radius $R$, we have (e.g., Van Hoolst and Dehant, 2002), correct up to the first order in the flattenings,

$$
\begin{aligned}
& A=I_{0}\left[1-\frac{1}{3} \alpha(R)-\frac{1}{2} \beta(R)\right], \\
& B=I_{0}\left[1-\frac{1}{3} \alpha(R)+\frac{1}{2} \beta(R)\right], \\
& C=I_{0}\left[1+\frac{2}{3} \alpha(R)\right],
\end{aligned}
$$

where

$I_{0}=\frac{8 \pi}{15} \rho R^{5}$.

As a consequence, the equatorial moment of inertia difference can be expressed as

$B-A=\frac{8 \pi}{15} \rho R^{5} \beta$.

For our models with homogeneous layers, the polar moment of inertia $C_{j}$ and the equatorial moment of inertia difference $B_{j}-A_{j}$ of layer $j$ can be derived by applying Eqs. (52)-(54) to two volumes considered to be of homogeneous density. First, 


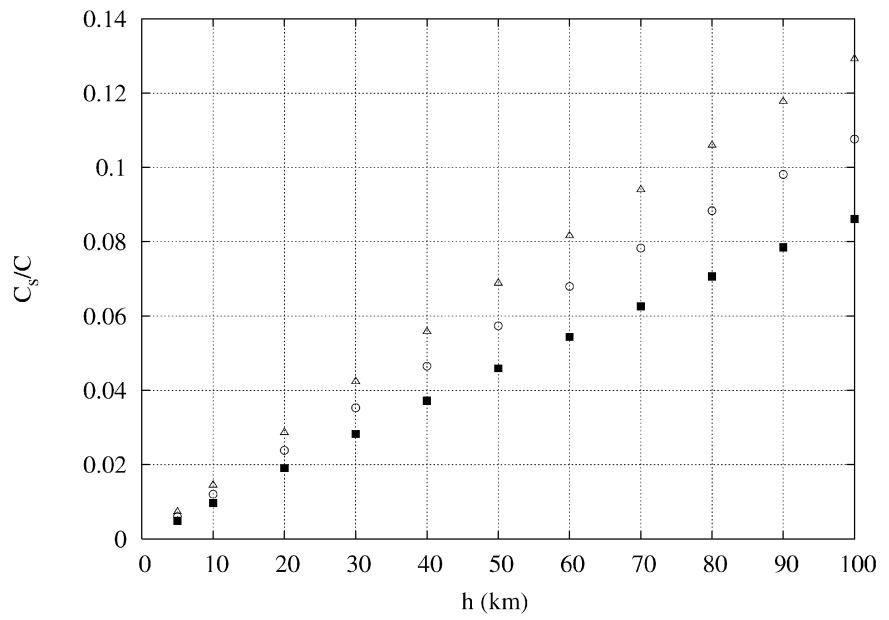

Fig. 6. Polar moment of inertia ratio of the icy shell and the whole satellite as a function of shell thickness for all our models of the interior structure of Europa. Models with the same shell thickness and density have almost equal ratio $C_{S} / C$ and cannot be distinguished in the figure. The difference in results at the same shell thickness is mainly due to the three different shell densities of the models. Shell densities are $800 \mathrm{~kg} \mathrm{~m}^{-3}$ (squares), $1000 \mathrm{~kg} \mathrm{~m}^{-3}$ (circles), and $1200 \mathrm{~kg} \mathrm{~m}^{-3}$ (triangles).

the volume between the center and the surface of layer $j$ is considered and secondly, the moments of inertia are calculated for the volume below layer $j$. Both volumes are considered to have the constant density $\rho_{j}$ of layer $j$. By taking the difference of the results, we then have

$$
\begin{aligned}
& C_{j}=\frac{8 \pi}{15} \rho_{j}\left[r_{0, j}^{5}\left(1+\frac{2}{3} \alpha_{j}\right)-r_{0, j-1}^{5}\left(1+\frac{2}{3} \alpha_{j-1}\right)\right], \\
& B_{j}-A_{j}=\frac{8 \pi}{15} \rho_{j}\left[r_{0, j}^{5} \beta_{j}-r_{0, j-1}^{5} \beta_{j-1}\right] .
\end{aligned}
$$

Here, $\alpha_{j}$ and $\beta_{j}$ are the polar flattening and equatorial flattening of the outer surface of layer $j$, respectively. The index $j$ increases with increasing distance of the layer $j$ to the center of the satellite. For the innermost layer containing the center, Eqs. (52) and (54) can be used directly. The polar moment of inertia and equatorial moment of inertia difference of the shell and solid interior are shown in Figs. 6 and 7.

\section{Results}

The amplitudes of the forced longitudinal libration at 3.55 days of the icy shell of Europa for our models of internal structure are calculated in the rigid approximation, for the decoupled shell, and for the libration model with internal gravitational coupling by using Eqs. (5), (7), (27) and (28), respectively. The relative differences with respect to the results of a direct numerical integration of the governing equations (1), (6), (19) and (20) is below $10^{-5}$ in all cases. We express the libration amplitude as a surface displacement at the equator corresponding to the angular displacement of the longest axis with respect to its position for a constant rotation rate of Europa.

Rigid librations are a good approximation for the librations of Europa if it does not have a subsurface ocean. The rigid libration amplitude has a mean value of $133.9 \mathrm{~m}$ at the equator and varies by at most $0.16 \%$ for our interior structure models. The

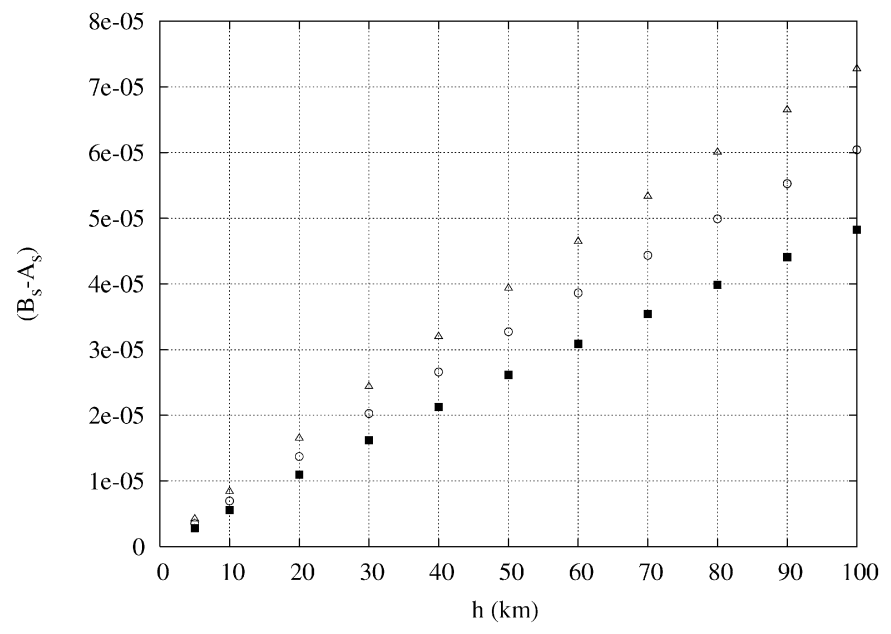

Fig. 7. Normalized equatorial moment of inertia difference of the icy shell $\left(B_{S}-A_{S}\right) / M_{E} R^{2}$ as a function of shell thickness $h$ for all our models of the interior structure of Europa. Shell densities are $800 \mathrm{~kg} \mathrm{~m}^{-3}$ (squares), $1000 \mathrm{~kg} \mathrm{~m}^{-3}$ (circles), and $1200 \mathrm{~kg} \mathrm{~m}^{-3}$ (triangles).

small variation is due to the small differences in the moments of inertia between the models. The amplitude is somewhat larger than the value of $112 \mathrm{~m}$ given by Comstock and Bills (2003) as a result of the different values of $(B-A) / C$ used. Comstock and Bills (2003) did not use the Radau equation and assumed Europa to have a uniform density.

As the shell polar moment of inertia is between about 7 and 200 times smaller than the polar moment of inertia of Europa (Fig. 6) for our interior structure models, the rotational inertia of a rotationally decoupled shell to an external torque is much weaker than for a rigid model, and the libration amplitude of the shell is proportionally larger and between about 1 and $27 \mathrm{~km}$ (Fig. 8 and Eq. (7)). Even larger values are obtained as a result of a near resonance between the orbital frequency and the frequency of the free shell libration for some models. The rigid libration amplitudes do not show any effect of a resonance because the free period for the rigid models, about 52.7 days, is too far from the forced period of 3.55 days. For models with thin shells, the frequency of the free libration of the icy shell can be much larger than the free frequency of a rigid Europa model because of the smaller polar moment of inertia $C_{s}$ compared to $C$ (see Eq. (8) and Fig. 6). The free shell libration period of our models varies between about 3.7 and 20 days. The smallest free period is very close to the forcing period of 3.55 days, leading to a resonant amplification of the forced libration. The corresponding interior structure model has a shell thickness of $5 \mathrm{~km}$ and a shell density of $800 \mathrm{~km} \mathrm{~m}^{-3}$. The resonance is only important for our models with a shell thickness of $5 \mathrm{~km}$. An exact resonance can be obtained for thin shells with $C_{s} / C=\left(\omega_{f} / n\right)^{2}=4.6 \times 10^{-3}$, which corresponds to shell thicknesses of somewhat less than $5 \mathrm{~km}$.

The large libration of a decoupled shell is clearly unrealistic as shown by the much smaller amplitude of libration when gravitational coupling is included. Fig. 9 represents the forced libration amplitude of the icy shell with internal gravitational coupling as a function of the ratio $\left(B_{S}-A_{s}\right) / C_{S}$. The amplitude is 6 to $14 \%$ larger than for the rigid libration applicable to Eu- 


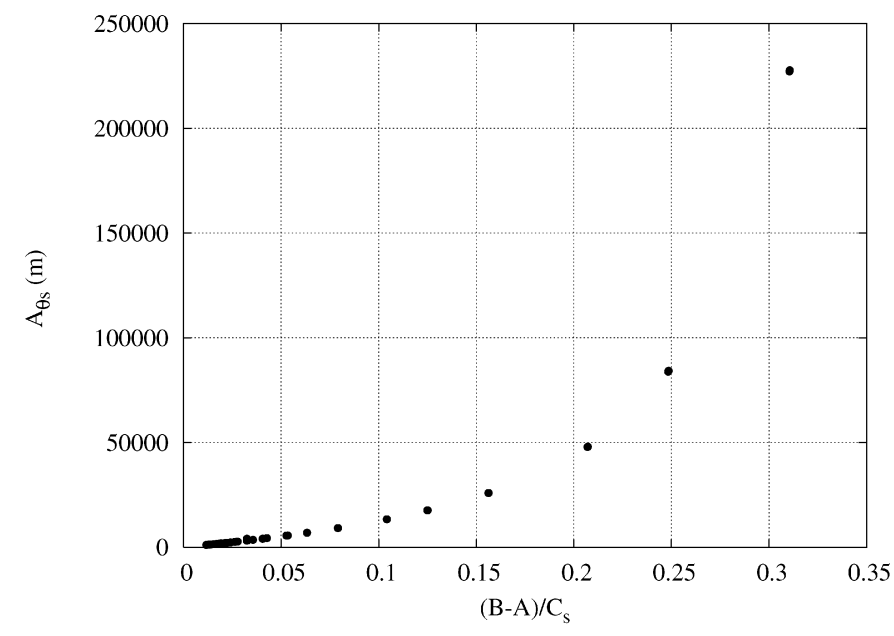

Fig. 8. Amplitude of the rotationally decoupled shell libration as a function of the inertia ratio $(B-A) / C_{S}$ for all our models of the interior structure of Europa.

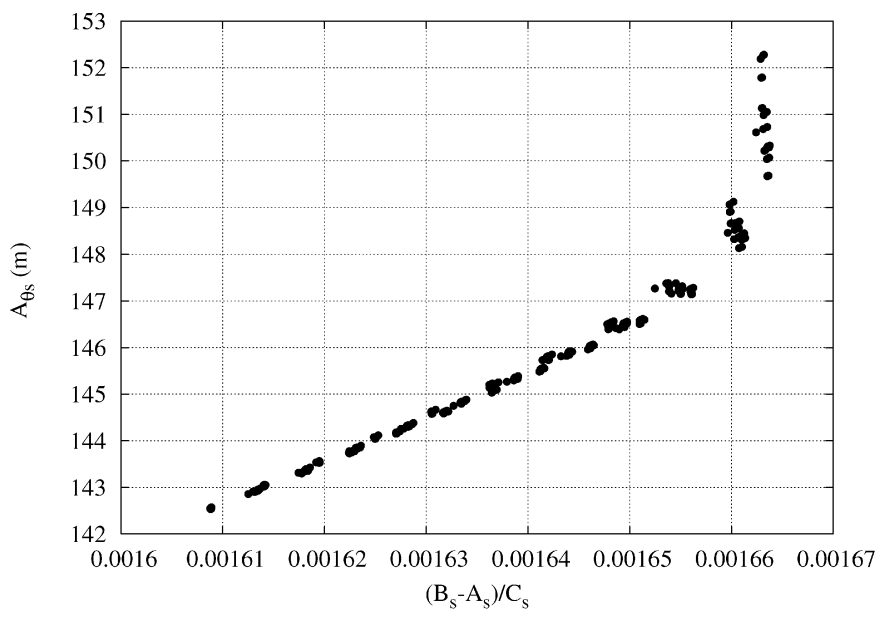

Fig. 9. Amplitude of the shell libration as a function of the moment of inertia ratio $\left(B_{S}-A_{S}\right) / C_{S}$ for all our models of the interior structure of Europa.

ropa models without ocean. This result shows that the shell and interior libration are strongly coupled by the gravitational force. The larger libration amplitude can be understood by noting that in the limit case of complete coupling between the shell and the solid interior the total polar moment of inertia of the shell and the interior is still somewhat smaller than the polar moment of inertia of the whole satellite, which includes the ocean. Therefore, even in that case, the rotational inertia would be smaller and the libration larger than for a rigid model.

Because of the amplitude difference with the rigid amplitude, libration observations can be used to determine the existence of a subsurface ocean, provided that the libration amplitude can be measured with a precision of a few meters and that the rigid libration amplitude can be calculated with at least a similar precision. The latter requires the knowledge of $(B-A) / C$ at the percent level, which is currently almost satisfied and will certainly be obtained with a future mission to Europa. A libration measurement precision of a few meters could also be obtained with a mission to Europa, as currently studied and proposed to both NASA and ESA. With only 15

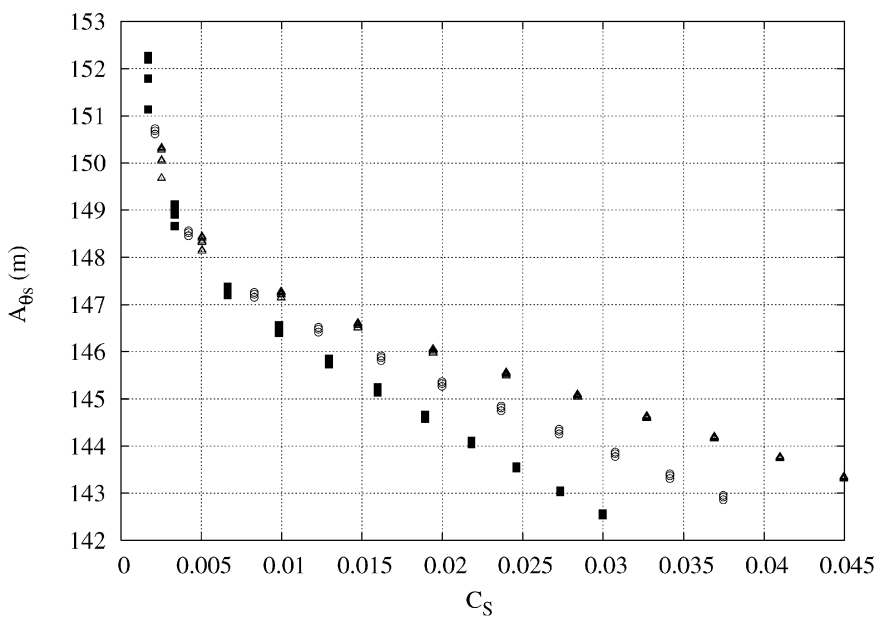

Fig. 10. Amplitude of the shell libration as a function of the normalized polar moment of inertia of the icy shell $C_{S} / M_{E} R^{2}$ for all our models of the interior structure of Europa. Shell densities are $800 \mathrm{~kg} \mathrm{~m}^{-3}$ (squares), $1000 \mathrm{~kg} \mathrm{~m}^{-3}$ (circles), and $1200 \mathrm{~kg} \mathrm{~m}^{-3}$ (triangles).

days of radio tracking data to a Europa orbiter, Wu et al. (2001) estimate a libration uncertainty of some tens of meters. With a longer data set of 2 months, the predicted lifetime of a Europa orbiter under study, an uncertainty of $10 \mathrm{~m}$ or less seems feasible. Librations can also be measured, with similar precisions, by taking multiple high-resolution camera images or laser altimeter measurements of selected geological features at different times, as planned for the MESSENGER and BepiColombo missions to measure the libration of Mercury (Solomon et al., 2001; Milani et al., 2001). With a lander on Europa's surface, an order of magnitude improvement can be expected with respect to libration estimates from an orbiter only, corresponding to a $1 \mathrm{~m}$ uncertainty on the shell libration.

As can be seen in Fig. 9, the shell libration amplitude varies by about $7 \%$ from about 142 to $152 \mathrm{~m}$ for different interior models. Therefore, information on the interior of Europa can be obtained from the forced libration amplitude, provided that sufficiently accurate measurements can be made. In particular, the shell moment of inertia ratio $\left(B_{s}-A_{s}\right) / C_{s}$ can be determined accurately from libration observations since the libration amplitude is almost linear in $\left(B_{s}-A_{s}\right) / C_{s}$ (see Fig. 9). This linearity is essentially due to the external gravitational torque as for the two other libration models. For the largest moment of inertia ratios, equivalent to the smallest polar moment of inertia of the shell, the libration amplitude increases sharply. This increase is most likely due to a resonance with one of the free periods and will be studied in detail in a subsequent manuscript. Here, we concentrate on the linear regime.

The geophysically most important factor in the ratio $\left(B_{s}-\right.$ $\left.A_{s}\right) / C_{s}$ is the denominator $C_{s}$. Fig. 10 shows a decreasing trend of the libration amplitude of the shell with increasing polar moment of inertia of the shell of roughly $140 \mathrm{~m} / M_{E} R^{2}$. Variations due to other interior structure parameters such as the density and radius of the other layers are superposed on this trend and can only be as large as $2 \mathrm{~m}$ for the thickest shells. The internal structure parameter that causes most of these deviations from a strictly linear behavior is the density of the shell (see Fig. 10). 


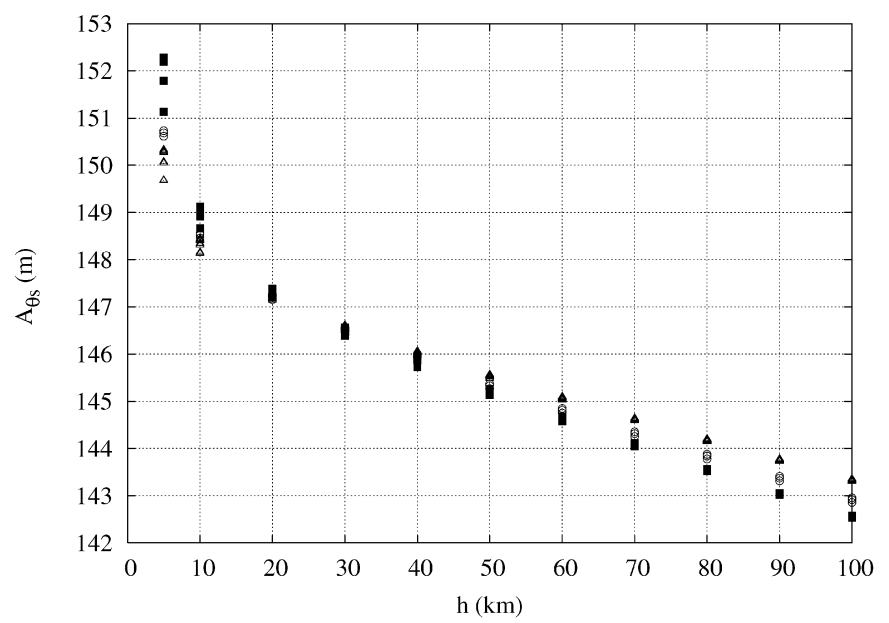

Fig. 11. Amplitude of the shell libration as a function of the thickness $h$ of the icy shell for all our models of the interior structure of Europa. Shell densities are $800 \mathrm{~kg} \mathrm{~m}^{-3}$ (squares), $1000 \mathrm{~kg} \mathrm{~m}^{-3}$ (circles), and $1200 \mathrm{~kg} \mathrm{~m}^{-3}$ (triangles).

Because we assumed a rather large range of shell density values in our models, the deviation from a strictly linear behavior could be somewhat less than indicated in Fig. 10. If libration amplitude observations are interpreted in terms of the polar moment of inertia of the icy shell, these variations imply an uncertainty on $C_{s} / M_{E} R^{2}$ of \pm 0.007 . Fig. 11 shows that the libration amplitude can also be interpreted directly in terms of the thickness of the icy shell. The amplitude decreases almost linearly with increasing shell thickness, with limited variation due to other interior structure parameters (see Fig. 11). For models with shell thickness larger than $10 \mathrm{~km}$, the libration amplitude shows a linearly decreasing trend of about $-0.055 \mathrm{~m} / \mathrm{km}$. An observational error of $1 \mathrm{~m}$ on the libration amplitude will therefore result in a uncertainty on the shell thickness of $18 \mathrm{~km}$. Therefore, libration observations can be used to constrain the thickness of the icy shell of Europa, but very precise libration observations are required. The libration amplitude variations due to the other parameters, which we estimate at $0.3 \mathrm{~m}$ at a shell thickness of $30 \mathrm{~km}$ (see Fig. 11), imply an additional uncertainty on the estimation of the shell thickness of $3 \mathrm{~km}$. The different amplitudes at a given shell thickness are mainly due to the different shell density of the models as shown in Fig. 11.

Besides the shell libration, the libration of the interior can also be used to constrain Europa's interior (see Fig. 12). The interior libration amplitude increases with increasing $\left(B_{i}-\right.$ $\left.A_{i}\right) / C_{i}$, similarly as observed for the shell libration, and varies between about 118 and $123 \mathrm{~m}$ (a relative difference of $4 \%$ ). The amplitude is particularly sensitive to the radius of the solid interior and is larger for larger radius of the interior (see Fig. 12). Although the relative range of interior libration amplitudes is smaller than for the shell, the interior libration may still be used to constrain the interior of Europa since, as shown by $\mathrm{Wu}$ et al. (2001), it can more accurately be estimated from radio tracking data than the shell libration. The librations can be determined from the time-variable gravity field of Europa, which can be estimated by radio tracking of a Europa orbiter.

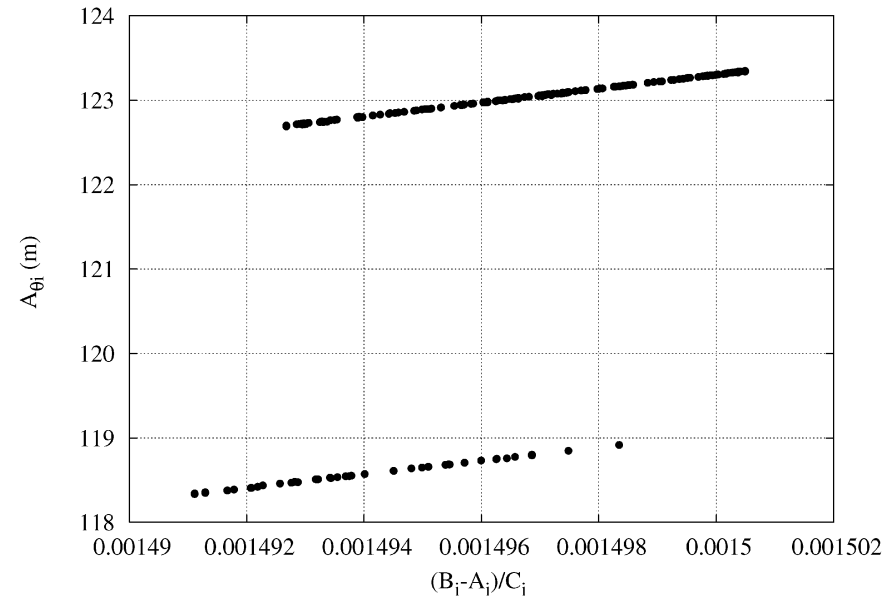

Fig. 12. Amplitude of the libration in longitude of the solid interior as a function of $\left(B_{i}-A_{i}\right) / C_{i}$ for all our models of the interior structure of Europa. The solutions are grouped around two mean values of 118.5 and $123 \mathrm{~m}$ depending on the radius of the mantle (1400 and $1450 \mathrm{~km})$.

\section{Discussion and conclusion}

Previous studies of the librations of Europa have assumed that the satellite responds rigidly to the gravitational torque of Jupiter. We have studied differential librations of the shell and the solid interior when a subsurface ocean exists in Europa. We have shown that the icy shell cannot be considered to librate independently from the interior. The most important coupling between the different layers is gravitational coupling between the icy shell and the solid interior. We have derived an expression for this internal gravitational coupling and included it into the differential equations governing the librations of the icy shell and of the solid interior. Analytical solutions for the librations of these two layers have been derived by assuming the librations to be small.

The main geophysical parameters for the librations are the polar moments of inertia of the layers, which are a measure of their resistance to rotational forcing, and the equatorial flattenings or equatorial moment of inertia differences, which determine the external gravitational torque of Jupiter on the layers and the internal gravitational coupling of the icy shell and the solid interior. In order to investigate the use of libration observations for the determination of the existence and properties of a putative subsurface ocean, we have calculated libration amplitudes for a range of interior structure models of Europa.

First, spherically symmetric models have been constructed from three observational constraints: the mass, radius, and mean moment of inertia. The latter quantity is determined from the observed $C_{22}$ (or $J_{2}$ ) gravity coefficients and the mean rotation rate by using the Radau equation. This equation is well known for bodies flattened by rotation, and we have extended and modified it to synchronously rotating satellites in hydrostatic equilibrium that are deformed by rotation and static tides. Secondly, we have calculated the permanent rotational and tidal internal deformation using Clairaut's theory and have determined the geophysical parameters of the libration for our Europa models. 
With an ocean, the forced libration amplitude is 6 to $14 \%$, or 8 to $18 \mathrm{~m}$, larger than for the rigid libration applicable to Europa models without ocean. Therefore, libration observations can be used to determine the existence of a subsurface ocean provided that the libration amplitude can be measured with a precision of less than $10 \mathrm{~m}$, which seems feasible with current technology.

Information on the thickness of the icy shell is also included in the libration amplitude, but this parameter is more difficult to retrieve. For Europa models with a subsurface ocean, the libration amplitude shows an increasing trend by about $6 \%$ from 142 to $149 \mathrm{~m}$ with decreasing shell thickness of our models, which can be used to estimate the shell thickness. However, the dependence is not very strong and a $1 \mathrm{~m}$ error on the libration amplitude corresponds to an uncertainty on the shell thickness of $18 \mathrm{~km}$. Additional information on the interior, in particular the density and radius of the solid interior, could be obtained from observations of the libration of the solid interior, and could improve this situation.

The gravitational torque of Jupiter on the permanent deformation of Europa, as considered in this study, is not the only cause of rotation variations of Europa. Zonal tides due to the gravitational force of Jupiter change the polar moment of Europa and therefore also the rotation. The variation in polar moment of inertia can be determined by using the definition of the tidal Love number $k_{2}$, which states that the change in the external gravitational potential at the surface due to mass redistribution in Europa induced by the tidal potential is equal to the product of the Love number and the tidal potential. Because the zonal degree-two time-variable tidal potential at the surface can be expressed as

$U_{t}^{2,0}(R, \phi, t)=-\frac{3}{2} \omega^{2} R^{2} e P_{2}(\cos \phi) \cos M$,

it then follows that the tidal change in the gravitational $J_{2}$ coefficient is given by

$\delta J_{2}=\frac{3}{2} k_{2} q e \cos M$.

Since the mean moment of inertia is conserved under these tidal deformations (Rochester and Smylie, 1974), we then have the following change in the polar moment of inertia due to zonal tides

$\delta C=k_{2} q e M_{E} R^{2} \cos M$.

In the case that Europa has no subsurface ocean, the rotational effect of the zonal tides can be estimated by calculating the ratio of the additional term $\omega \delta \dot{C}$ due to the tides and the main term $C \ddot{\theta}$ in the governing equation (1) for libration. This ratio

$\frac{k_{2} q e}{A_{\theta}} \frac{M_{E} R^{2}}{C} \approx 0.038$

is small, suggesting that the effect of zonal tides on libration is on the order of a few percent and does not change the main conclusions of this study.

A potential problem of the use of libration observations to determine the existence of a subsurface ocean and to constrain the shell thickness is that Europa might not be in a fully relaxed hydrostatic equilibrium. In that case, the Radau equation is not strictly valid, and the polar moment of inertia $C$ could be slightly different. The relative difference with the hydrostatic value is expected to be smaller than $1 \%$, and could be estimated once $J_{2}$ is determined independently from $C_{22}$ from spacecraft flybys. Moreover, the internal flattening cannot be calculated from Clairaut's equation in that case. It is difficult to estimate the deviations of the internal flattening from the hydrostatic values, these would depend among other things on possible convection in the solid interior, but they are expected to be small with respect to the rotational and tidal deformations for the rapidly rotating Europa. Verification of the hydrostatic relation $J_{2}=(10 / 3) C_{22}$ by future space measurements could be used as an indication of the correctness of the hydrostatic assumption, although it cannot prove the validity of the approximation.

\section{Acknowledgments}

Xiaoping Wu and David O'Brien are kindly thanked for constructive reviews that helped to improve this manuscript. This work was financially supported by the Belgian PRODEX program managed by the European Space Agency in collaboration with the Belgian Federal Science Policy Office. N.R. acknowledges the support of a Prodex/ESA contract C90253 at the FUNDP.

\section{Appendix A. An alternative form of the Radau equation extended to synchronously rotating satellites}

A somewhat different form of the extended Radau equation for a synchronously rotating satellite, deformed by rotation and tides, can be obtained by expressing $J_{2}$ in Eq. (47) in terms of the fluid Love number $k_{f}$, which describes the reaction of Europa to a perturbing potential after all viscous stresses have relaxed (Munk and MacDonald, 1960). By using MacCullagh's formula for the gravitational potential of a nearly spherical body and identifying the degree-two, order-zero term and the degreetwo, order-two term of the gravitational potential field of Europa with the corresponding terms in $k_{f}\left[U_{r}(\vec{r})+U_{t}(\vec{r})\right]$, we have

$$
\begin{aligned}
& J_{2}=\frac{5}{6} k_{f} q, \\
& C_{22}=\frac{1}{4} k_{f} q
\end{aligned}
$$

(e.g., Rappaport et al., 1997). Substitution of Eq. (A.1) into Eq. (47) gives

$\frac{I}{M_{E} R^{2}}=\frac{2}{3}\left[1-\frac{2}{5}\left(\frac{4-k_{f}}{1+k_{f}}\right)^{1 / 2}\right]$.

In contrast to the expressions (42) and (47) in terms of $\alpha(R)$ or $J_{2}$ and $q$, this expression takes the same form as for a satellite that is only deformed by rotation. It must be remarked though, that the relation between $J_{2}$ and $k_{f}$ is different, and therefore the numerical value of $k_{f}$ associated to the observed value of $J_{2}$ is also different from the value in the case of rotational deformation only. In the classical derivation of this equation (e.g., 
Hubbard, 1984), only rotational deformation is taken into account.

By using Eqs. (45), (46), (A.1), and (A.2), we also have for the surface flattenings

$\alpha(R)=\frac{5}{4} q\left(1+k_{f}\right)$,

and

$\beta(R)=\frac{3}{2} q\left(1+k_{f}\right)$,

which are consistent with the ratio $5 / 6$ between $\alpha$ and $\beta$ throughout the satellite (see Section 3.4).

\section{References}

Anderson, J.D., Schubert, G., Jacobson, R.A., Lau, E.L., Moore, W.B., Sjogren, W.L., 1998. Europa's differentiated internal structure: Inferences from four Galileo encounters. Science 281, 2019-2022.

Billings, S.E., Kattenhorn, S.A., 2005. The great thickness debate: Ice shell thickness models for Europa and comparisons with estimates based on flexure at ridges. Icarus 177, 397-412.

Buffett, B.A., 1996. Gravitational oscillations in the length of day. Geophys. Res. Lett. 23 (17), 2279-2282.

Carr, M.H., and 21 colleagues, 1998. Evidence for a subsurface ocean on Europa. Nature 391, 363-365.

Cayley, A., 1861. Tables of the developments of functions in the theory of elliptic motion. Mem. R. Astron. Soc. 29, 191-306.

Castillo, J., Mocquet, A., Sotin, C., 2000. Détecter la présence d'un océan dans Europe à partir de mesures altimétriques et gravimétriques. C. R. Acad. Sci. Paris 330, 659-666.

Comstock, R.L., Bills, B.G., 2003. A Solar System Survey of forced librations in longitude. J. Geophys. Res. 108 (E9), doi:10.1029/2003JE00210. 5100.

Darwin, G.H., 1899. The theory of the figure of the Earth carried to the second order of small quantities. Mon. Not. R. Astron. Soc. 60, 82-124.

Denis, C., Amalvict, M., Rogister, Y., Tomecka-Suchoń, S., 1998. Methods for computing internal flattening, with applications to the Earth's structure and geodynamics. Geophys. J. Int. 132, 603-642.

Goldreich, P., Peale, S., 1966. Spin-orbit coupling in the Solar System. Astron. J. 71, 425-438.

Hansen, P.A., 1855. Abhandlungen der Mathematisch-Physischen Class der Königlich Sächsischen Gesellschaft der Wissenschaften, Leipzig, vol. 2, pp. 515-523.

Henrard, J., 2005. The rotation of Europa. Celest. Mech. Dynam. Astron. 91. pp. 131-149.

Hubbard, W.B., 1984. Planetary Interiors. Van Nostrand, New York.

Hughes, S., 1981. The computation of tables of Hansen coefficients. Celest. Mech. 25, 101-107.

Hussmann, H., Sohl, F., Spohn, T., 2006. Subsurface oceans and deep interiors of medium-sized outer planet satellites and large trans-neptunian objects. Icarus 185, 258-273.

Jeffreys, H., 1952. The Earth: Its Origin, History and Physical Constitution. Cambridge Univ. Press, Cambridge.

Kargel, J.S., Kaye, J.Z., Head, J.W., Marion, G.M., Sassen, R., Crowley, J.K., Ballesteros, O.P., Grant, S.A., Hogenboom, D.L., 2000. Europa's crust and ocean: Origin, composition, and the prospects for life. Icarus 148, 226-265.

Khurana, K.K., Kivelson, M.G., Stevenson, D.J., Schubert, G., Russell, C.T., Walker, R.J., Polanskey, C., 1998. Induced magnetic fields as evidence for subsurface oceans in Europa and Callisto. Nature 395, 777-780.

Kivelson, M.G., Khurana, K.K., Stevenson, D.J., Bennett, L., Joy, S., Russell, C.T., Walker, R.J., Zimmer, C., Polanskey, C., 1999. Europa and Callisto: Induced or intrinsic fields in a periodically varying plasma environment. J. Geophys. Res. 104, 4609-4626.

Kivelson, M.G., Khurana, K.K., Russell, C.T., Volwerk, M., Walker, R.J., Zimmer, C., 2000. Galileo magnetometer measurements: A stronger case for a subsurface ocean at Europa. Science 289, 1340-1343.
Kovack, R.L., Chyba, C.F., 2001. Seismic detectability of a subsurface ocean on Europa. Icarus 150, 279-287.

Lee, S., Zanolin, M., Thode, A.M., Pappalardo, R.T., Makris, N.C., 2003. Probing Europa's interior with natural sound sources. Icarus 165, 144-167.

McCord, T.B., and 11 colleagues, 1998. Salts on Europa's surface detected by Galileo's Near Infrared Mapping Spectrometer. Science 280, 12421245.

Milani, A., Rossi, A., Vokrouhlicky, D., Villani, D., Bonnano, C., 2001. Gravity field and rotation state of Mercury from the BepiColombo Radio Science Experiments. Planet. Space Sci. 49, 1579-1596.

Moritz, H., 1990. The Figure of the Earth. Herbert Wichmann Verlag GmbH, Karlsruhe.

Moritz, H., Mueller, I.I., 1987. Earth Rotation: Theory and Observation. Ungar, New York.

Munk, W.H., MacDonald, G.J.F., 1960. The Rotation of the Earth: A Geophysical Discussion. Cambridge Univ. Press, Cambridge.

Murray, C.D., Dermott, S.F., 2000. Solar System Dynamics. Cambridge Univ. Press, Cambridge.

Pappalardo, R.T., and 31 colleagues, 1999. Does Europa have a subsurface ocean? Evaluation of the geological evidence. J. Geophys. Res. 104, 2401524056.

Peale, S.J., Phillips, R.J., Solomon, S.C., Smith, D.E., Zuber, M.T., 2002. A procedure for determining the nature of Mercury's core. Meteorit. Planet. Sci. 37, 1269-1283.

Plummer, H.C., 1918. An Introductory Treatise on Dynamical Astronomy. Cambridge Univ. Press, Cambridge.

Radau, R., 1885. Sur la loi des densités à l'intérieur de la Terre. C. R. Acad. Sci. Paris 100, 972-974.

Rambaux, N., Van Hoolst, T., Dehant, V., Bois, E., 2007. Inertial core-mantle coupling and libration of Mercury. Astron. Astrophys. 468, 711-719.

Rappaport, N., Bertotti, B., Giampieri, G., Anderson, J.D., 1997. Doppler measurements of the quadrupole moments of Titan. Icarus 126, 313-323.

Rochester, M.G., Smylie, D.E., 1974. On changes in the trace of the Earth's inertia tensor. J. Geophys. Res. 79, 4948-4951.

Ross, M., Schubert, G., 1987. Tidal heating in an internal ocean model of Europa. Nature 325, 132-134.

Schenk, P., 2001. Topographic variability on Europa from Galileo stereo images. Lunar Planet. Sci. XXXII. Abstract 2078.

Sohl, F., Spohn, T., Breuer, D., Nagel, K., 2002. Implications from Galileo observations on the interior structure and chemistry of the Galilean satellites. Icarus 157, 104-119.

Solomon, S.C., and 20 colleagues, 2001. The MESSENGER mission to Mercury: Scientific objectives and implementation. Planet. Space Sci. 49, 14451465.

Sotin, C., Head, J.W., Tobie, G., 2002. Europa: Tidal heating of upwelling thermal plumes and the origin of lenticulae and chaos melting. Geophys. Res. Lett. 29 (8), doi:10.1029/2001GL013844.

Spohn, T., Schubert, G., 2003. Oceans in the icy Galilean satellites of Jupiter? Icarus 161, 456-467.

Squyres, S.W., Reynolds, R.T., Cassen, P.M., 1983. Liquid water and active resurfacing on Europa. Nature 301, 225-226.

Szeto, A.M.K., Xu, S., 1997. Gravitational coupling in a triaxial ellipsoidal Earth. J. Geophys. Res. 102, 27651-27657.

Van Hoolst, T., 2007. Rotation of Terrestrial Planets: Treatise on Geophysics, vol. 10. Planets and Moons. Elsevier, in press.

Van Hoolst, T., Dehant, V., 2002. Influence of triaxiality and second-order terms in flattenings on the rotation of terrestrial planets. I. Formalism and rotational normal modes. Phys. Earth Planet. Int. 134, 17-33.

Wahr, J.M., Zuber, M.T., Smith, D.E., Lunine, J.I., 2006. Tides on Europa, and the thickness of Europa's icy shell. J. Geophys. Res. 111, doi:10.1029/ 2006JE002729. E12005.

Wu, X., Bar-Sever, Y.E., Folkner, W.M., Williams, J.G., Zumberge, J.F., 2001. Probing Europa's hidden ocean from tidal effects on orbital dynamics. Geophys. Res. Lett. 28 (11), 2245-2248.

Xu, S., Crossley, D., Szeto, A.M.K., 2000. Variations in length of day and inner core differential rotation from gravitational coupling. Phys. Earth Planet. Int. 117, 95-110. 